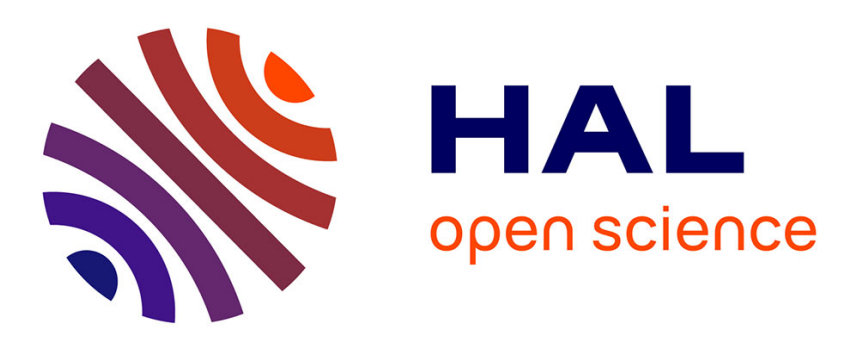

\title{
Parallel Transport of Surface Deformations from Pole Ladder to Symmetrical Extension
}

Shuman Jia, Nicolas Duchateau, Pamela Moceri, Maxime Sermesant, Xavier

Pennec

\section{- To cite this version:}

Shuman Jia, Nicolas Duchateau, Pamela Moceri, Maxime Sermesant, Xavier Pennec. Parallel Transport of Surface Deformations from Pole Ladder to Symmetrical Extension. Shape in Medical Imaging. ShapeMI 2018., Sep 2018, Granada, Spain. pp.116-124, 10.1007/978-3-030-04747-4_11. hal01860274

\section{HAL Id: hal-01860274 \\ https://hal.inria.fr/hal-01860274}

Submitted on 23 Aug 2018

HAL is a multi-disciplinary open access archive for the deposit and dissemination of scientific research documents, whether they are published or not. The documents may come from teaching and research institutions in France or abroad, or from public or private research centers.
L'archive ouverte pluridisciplinaire HAL, est destinée au dépôt et à la diffusion de documents scientifiques de niveau recherche, publiés ou non, émanant des établissements d'enseignement et de recherche français ou étrangers, des laboratoires publics ou privés. 


\title{
Parallel Transport of Surface Deformations from Pole Ladder to Symmetrical Extension
}

\author{
Shuman $\mathrm{Jia}^{1}$, Nicolas Duchateau ${ }^{2}$, Pamela Moceri ${ }^{1,3}$, Maxime Sermesant ${ }^{1}$ and \\ Xavier Pennec ${ }^{1}$ \\ 1 Université Côte d'Azur, Epione Project, Inria Sophia Antipolis, France \\ 2 Creatis, CNRS UMR5220, INSERM U1206, Université Lyon 1, France \\ 3 Hôpital Pasteur, CHU de Nice, France
}

\begin{abstract}
Cardiac motion contains information underlying disease development, and complements the anatomical information extracted for each subject. However, normalization of temporal trajectories is necessary due to anatomical differences between subjects. In this study, we encode inter-subject shape variations and temporal deformations in a common space of diffeomorphic registration. They are parameterized by stationary velocity fields. Previous normalization algorithms applied in medical imaging were first order approximations of parallel transport. In contrast, pole ladder was recently shown to be a third order scheme in general affine connection spaces and exact in one step in affine symmetric spaces. We further improve this procedure with a more symmetric mapping scheme, which relies on geodesic symmetries around mid-points. We apply the method to analyze cardiac motion among pulmonary hypertension populations. Evaluation is performed on a 4D cardiac database, with meshes of the right-ventricle obtained by commercial speckle-tracking from echo-cardiogram. We assess the stability of the algorithms by computing their numerical inverse error. Our method turns out to be very accurate and efficient in terms of compactness for subspace representation.
\end{abstract}

\section{Introduction}

Analyzing cardiac function, notably myocardial deformation along cardiac cycle, can reveal subtle changes underlying disease development, and therefore improve risk stratification for patients. However, temporal deformations (cyclical or longitudinal) are complex due to subject-specific anatomy. Normalization of these trajectories into a common reference frame is an essential prerequisite in statistical analysis $[4,1]$.

Nevertheless, it is a challenging task to construct a robust method for the normalization of temporal deformations, both theoretically and numerically. In cardiac imaging, the first step often includes segmenting the region of interest from the images, which generates its surface or volume as results. Then, to model the deformation between two shapes, depending on properties of the shapes, different non-rigid registration algorithms may be suitable in specific scenario. 

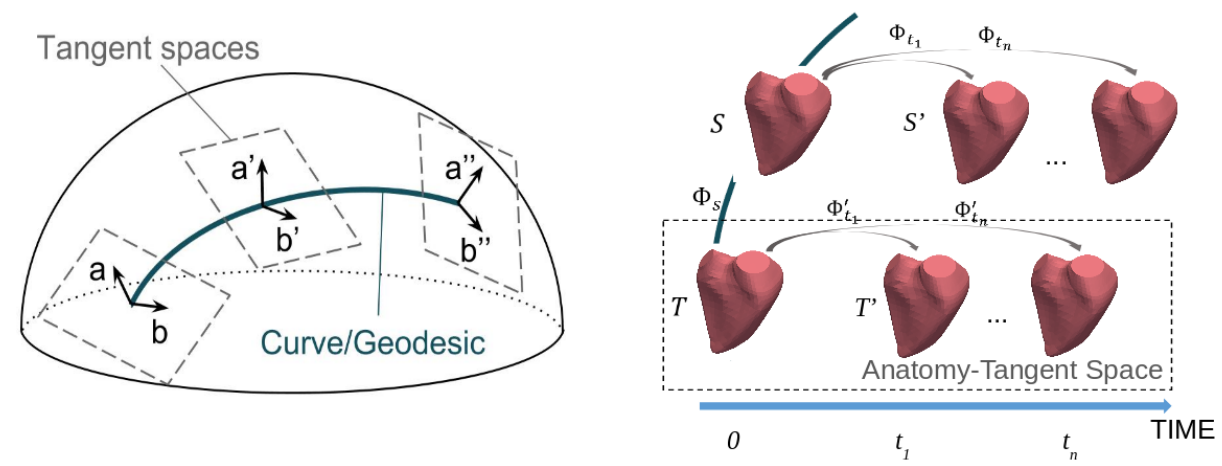

Fig. 1. Illustration of parallel transport of vectors $a$ and $b$ along a curve (left) and its application to cardiac imaging (right) with a focus on surfaces.

We choose to place our method in a space of diffeomorphic transformations. In particular, diffeomorphism provides invertible and folding-free registration results, which enables a symmetric encoding of deformation trajectories.

Given that diffeomorphisms are parameterized via flow of velocity fields, here stationary velocity field (SVF) [13], their normalization requires vector-based transport. In differential geometry, parallel transport specifies how to realize an infinitesimal transformation (a tangent vector) along a curve from one point to another of a manifold, as illustrated in Fig. 1(a). The concept was introduced in medical imaging by [14] and used for neuroimaging studies in [11]. To apply it to cardiac sequences, we need to:

- Parameterize the temporal deformations $\Phi_{t_{i}}$ from the subject-specific shape $S$ at baseline to its shape $S_{t_{i}}^{\prime}$ at time $t_{i}$ by SVF $v_{t_{i}}$; this step amounts to computing the logarithm of the deformations such that $\Phi_{t_{i}}=\exp \left(v_{t_{i}}\right)$, or to register $S$ to $S_{t_{i}}^{\prime}$.

- Transfer these SVFs along the inter-subject deformation geodesic $\Phi_{S}$ from the space of $S$ to a common geometry $T$, often called a template.

These steps lead to a representation of all the subject-specific deformations as infinitesimal deformations of the same anatomy. We can thus perform proper population comparison in terms of shape evolution along the cardiac cycle, as shown in Fig. 1(b).

Despite the fact that the definition of logarithm may be well developed in theory, implementations of its computation could be unstable due to numerical inconsistency. While computing registration/geodesics, the logarithms of surface deformations are only sparsely defined in the space (on the shape), which differs from image deformations. Careful numerical implementation of parallel transport algorithm is also needed.

Parallel transport methods in medical image analysis are currently based on ladders (like Schild's and pole ladders $[6,7]$ ) or on variations of Jacobi Fields $[14,8]$. These methods are first order approximation schemes which need to be 


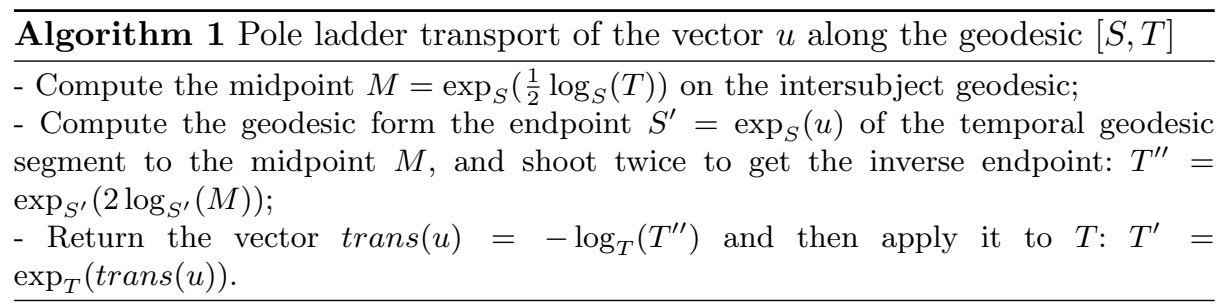

iterated along the curve. In contrast, pole ladder was recently discovered to be of third order in general affine connection spaces [10], which makes it a very attractive method to implement. In order to further improve the symmetry and thus the numerical stability with respect to the implementation of [7], our method relies on the definition of middle point and geodesic symmetry.

\section{Methodology}

\subsection{Pole Ladder}

Pole ladder is based on using the curve as the diagonal of the geodesic parallelogram to realize parallel transport (see Fig 2 and Algo. 1). Let $u$ be the initial tangent vector to the geodesic segment that encodes the temporal deformation $\exp _{S}(t u)(t \in[0,1])$. Originally, pole ladder computes a midpoint on the geodesic $[S, T]$ and expands twice the geodesic form the endpoint $S^{\prime}$ to the midpoint to obtain the point $T^{\prime \prime}$. In this paper, we propose to reformulate the doubling of the geodesics with a mid-point geodesic symmetry (Algo. 2). The mid-point $M$ of a geodesic segment $[S, T]$ is defined as $M=\gamma_{[S, T]}\left(\frac{1}{2}\right)$. The mappings from $M$ towards the two sides meet the definition of a geodesic symmetry: $\gamma_{[M, S]}(t)=-\gamma_{[M, T]}(t)$. Although this novel version of pole ladder is theoretically equivalent to the previous one, it is numerically more stable in our experiments. This may be emphasized by the fact that in medical imaging we are encoding tangent vectors with infinitesimal deformations and that changing the object which is deformed may have a drastic numerical impact.

\subsection{Numerical Accuracy}

Recent mathematical developments have enabled the numerical analysis of pole ladder in affine connection spaces with symmetric connections [10]. In Lie groups,

\footnotetext{
Algorithm 2 Mid-point symmetric pole ladder transport of the geodesic segment $\left[S, S^{\prime}\right]$ along the geodesic $[S, T]$

- Compute the midpoint $M=\gamma_{[S, T]}\left(\frac{1}{2}\right)$ on the intersubject geodesic;

- Compute the symmetric point $T^{\prime \prime}=\exp _{M}\left(-\log _{M}\left(S^{\prime}\right)\right)$ of $S^{\prime}$ with respect to $M$;

- Compute the symmetric point $T^{\prime}=\exp _{T}\left(-\log _{T}\left(T^{\prime \prime}\right)\right)$ of $T^{\prime \prime}$ with respect to $T$, and return the geodesic segment $\left[T, T^{\prime}\right]$.
} 


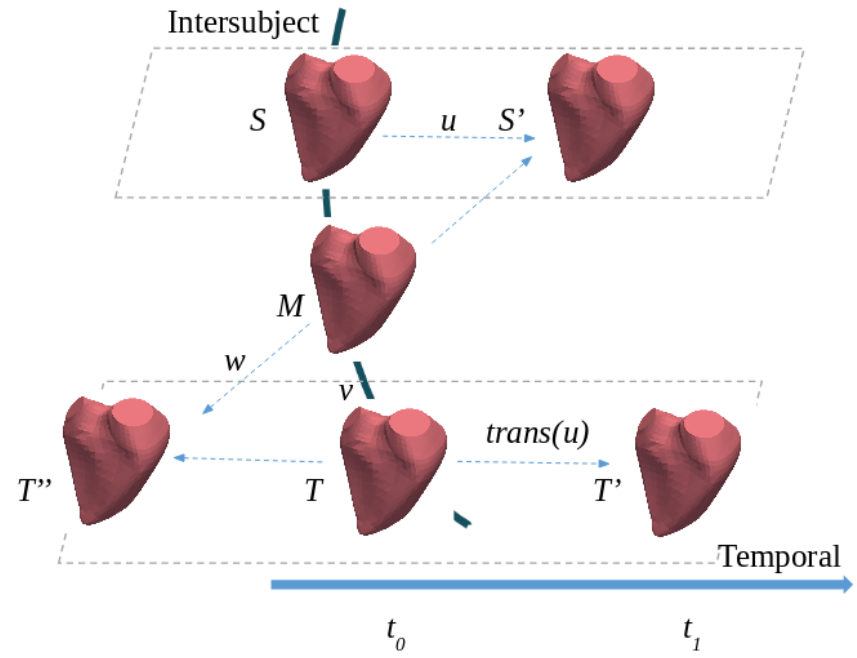

Fig. 2. The symmetry-based pole ladder structure.

the Baker-Campbell-Hausdorff (BCH) formula provides an expansion of the composition of two group exponentials in the Lie algebra: $B C H(v, u)=\log (\exp (v) \circ$ $\exp (u))$. This formula has been thoroughly used in registration algorithms [2] In general affine connection manifolds, a somewhat similar formula can be established based on the curvature instead of the Lie bracket [5]. The double exponential $\exp _{x}(v, u)=\exp _{y}\left(\Pi_{x}^{y} u\right)$ corresponds to a first geodesic shooting from the point $x$ along the vector $v$, followed by a second geodesic shooting from $y=\exp _{x}(v)$ along the parallel transport $\Pi_{x}^{y} u$ of the vector $u$. [5] has shown that the Taylor expansion of the $\log$ of this endpoint $h_{x}(v, u)=\log _{x}\left(\exp _{x}(v, u)\right)$ is:

$$
\begin{aligned}
h_{x}(v, u)= & v+u+\frac{1}{6} R(u, v) v+\frac{1}{3} R(v, u) u+\frac{1}{12} \nabla_{v} R(u, v) v+\frac{1}{24}\left(\nabla_{u} R\right)(u, v) v \\
& +\frac{5}{24}\left(\nabla_{v} R\right)(u, v) u+\frac{1}{12}\left(\nabla_{u} R\right)(u, v) u+O\left(\|u+v\|^{5}\right) .
\end{aligned}
$$

When applied to our reformulation of pole ladder using geodesic symmetry, we find that the error on one step of pole ladder to transport the vector $u$ along a geodesic segment of tangent vector $[S, T]=\left[\exp _{M}(-v), \exp _{M}(v)\right]$ (all quantities being parallel translated at the mid-point $M)$ is:

$\Pi_{T}^{M} \operatorname{trans}(u)-\Pi_{S}^{M} u=\frac{1}{12}\left(\left(\nabla_{v} R\right)(u, v)(5 u-2 v)+\left(\nabla_{u} R\right)(u, v)(v-2 u)\right)+O\left(\|v+u\|^{5}\right)$.

We see here that one single step of pole ladder is of order three, thus much more accurate than the other first order parallel transport schemes. Moreover, the fourth order error term vanishes in affine symmetric spaces since the curvature is covariantly constant in these spaces. In fact, one can actually prove 
that all error terms vanish in a convex normal neighborhood of an affine connection space: one step of pole ladder realizes an exact parallel transport (provided that geodesics and mid-points are computed exactly of course) [10]. This result makes pole ladder a very attractive scheme. In particular, Lie groups have a canonical symmetric space structure thanks to the symmetry $s_{g}(h)=g h^{-1} g$ for any elements $g, h$ of the Lie group. This is an affine structure which is generally not metric. The symmetry generates a canonical connection which is exactly the symmetric Cartan-Schouten connection. Since the geodesics of this connection going through identity are parameterized the flow of SVFs, we can conclude that pole ladder on SVFs is exact, at least theoretically.

\subsection{SVF-Based Transport}

In practice, we consider sparse point-to-point correspondences between two shapes (established here with the commercial speckle-tracking software) to compute diffeomorphic transformation. Thus, here the solution for the log is not unique and needs to be spatially regularized. Following [12], the sparse displacement field on the mesh vertices is interpolated to a dense image grid using with a standard thin-plate spline (TPS) kernel (stiffness of zero) [3]. The TPS fits the smooth surfaces and resistance in myocardial contraction. Then, a symmetric iterative procedure computes explicitly a SVF from the displacement field using $\mathrm{BCH}$ formula. The result gives a smooth SVF that linearly extends outside the grid borders.

The SVFs for temporal and intrasubject transformations are then used to compute the transported SVF to the template space using our symmetric pole ladder transport. In this context, we hypothesize that cardiac shape variations and temporal deformations can be efficiently modeled in a space of diffeomorphic transformations. The detailed shape variability that is lost during registration process will be transported. That is the reason why we report fiducial localisation error due to shape registration in Sec 3.

\subsection{Benchmark Transport Algorithm}

Simple transport, conjugate action, which has been tested for cardiac motion analysis $[4,1]$, serves as a benchmark. The conjugate action of two transformations is defined as $\operatorname{Conj}_{\Phi_{s}}\left(\Phi_{t}\right)=\Phi_{s} \Phi_{t} \Phi_{s}^{-1}$. Numerically, it can be computed as the solution of $\exp \left(\operatorname{trans}\left(v_{t}\right)\right)=\exp \left(v_{s}\right) \exp \left(v_{t}\right) \exp \left(-v_{s}\right)$ in the SVF setting using iterated $\mathrm{BCH}$ formulas.

\section{Experiments}

\subsection{Materials}

We assess our methods on a 4D cardiac database of right-ventricular (RV) meshes. The population includes 34 healthy subjects, and 104 subjects with 
Table 1. Numerical consistency of parallel transports. Fiducial localisation error due to shape registration (left) and to forward/backward transport (right) ( $\mu \pm \sigma$ in $m m$ ).

$\frac{\frac{\text { Registration error (FLE) }}{\text { SVF }}}{0.15 \pm 0.15}$

\begin{tabular}{cc}
$\frac{\text { Inverse Transport }}{\text { Pole ladder SVF Conjugate SVF }}$ \\
\hline $0.12 \pm 0.10$ & $0.22 \pm 0.12$ \\
\hline
\end{tabular}

pulmonary hypertension, which are divided into three classes according to the severity of the disease (New York Heart Association - NYHA, classes II, III and IV). The motion of the myocardium was given by point-to-point correspondences from commercial speckle-tracking of the RV from 3D trans-thoracic echocardiography (by 4D RV Function 2.0 software, TomTec Imaging Systems GmbH, Germany). For the acquisition of images, please refer to [9].

\subsection{Template Estimation and Registration}

The meshes were spatially aligned at baseline time, end-diastole (ED), using rigid deformations with the iterative closest point algorithm. Then for meshes of different instants but belonging to one subject, we preserved their relative position between cardiac sequences by applying to them the same rigid deformation. A template was built at baseline time ED as a mean shape in the healthy group using point distribution model.

Then we compute the deformation from each subject's shape at ED towards the template. The middle point on this geodesic serves as the midpoint for the two symmetric mappings in pole ladder to transport temporal deformations, $\left[M, S^{\prime}\right],\left[M, T^{\prime \prime}\right]$ and $\left[T, T^{\prime \prime}\right],\left[T, T^{\prime}\right]$, as shown in Fig 2 . The computation of SVFs is implemented in $\mathrm{C}++$.

\subsection{Consistency}

Inverse consistency was tested by transporting the velocity field corresponding to the end-diastole-end-systole (ED-ES) deformation forward along the geodesic encoding the subject-specific RV shape changes with respect to the template at $\mathrm{ED}$, and then backward along the reverse geodesic. If the numerical implementation is accurate, the result of the inverse transport should be exactly the original vector.

Consistency was quantified by the error between the final warped end-systole (ES) shape and the original subject's ES shape (Table 1). The errors should be compared to the mismatch of each registration method (fiducial localisation error of vertices). We can see that SVF pole ladder transport was very consistent.

\subsection{Application to the Statistical Modeling of Cardiac Deformation}

In this subsection, we model ED-ES deformation in the whole subgroup of healthy subjects using parallel transports. Similar to the first step described 


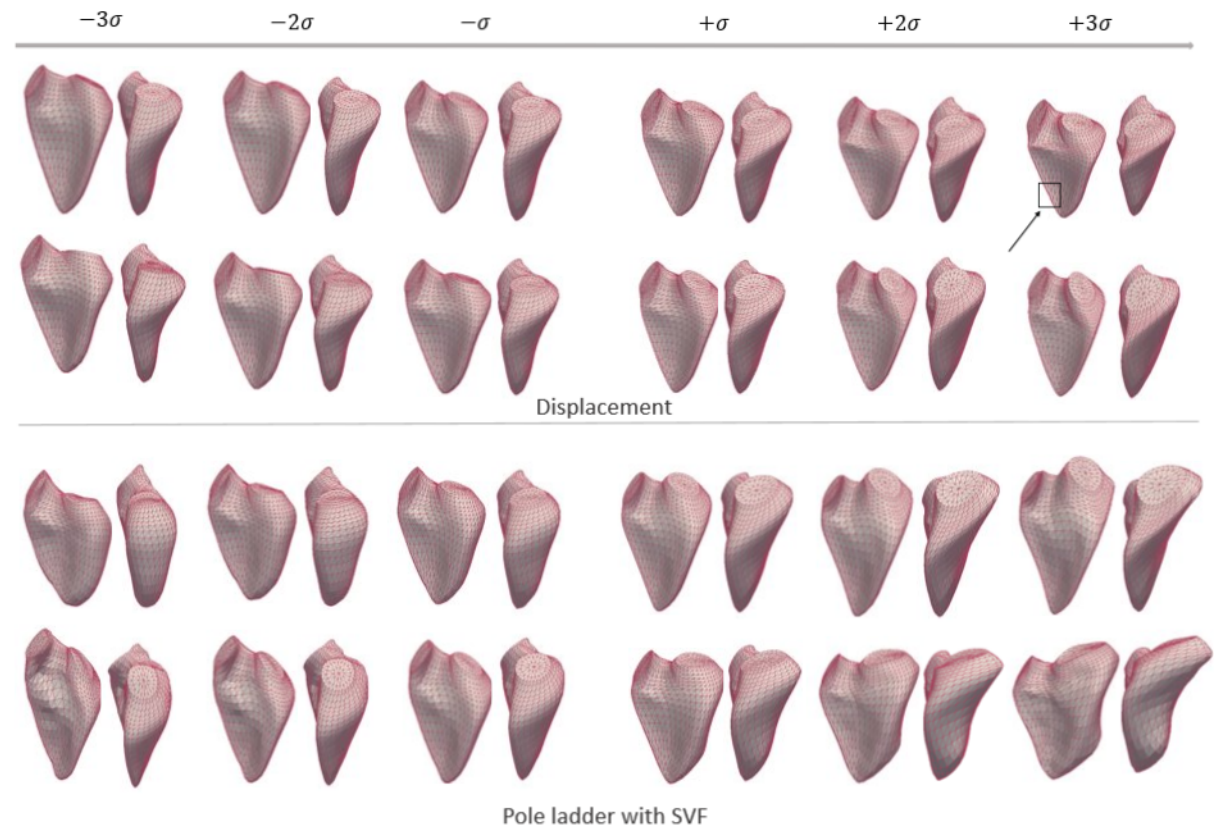

Fig. 3. Fist two modes of variation after applying PCA on the transported ED-ES changes, for the healthy subgroup. Comparison of point-to-point (top) and pole ladder with SVF (bottom). Surface folds appear at the arrow.

in the previous experiments, we transported the velocity fields associated to each subject's ED-ES changes to the template at ED. Then, we applied principal component analysis (PCA) on the transported velocity fields, and warped the template with resulting principal components to show the main modes of deformation among this subgroup. We also compared this with the PCA modes obtained from the point-to-point ED-ES displacement.

The first mode from point-topoint displacement accounts for the elongation (no relative displacement of the two valves), the second accounts for the vertical displacement and a slight orientation of the tricuspid valve. The first two modes from the pole ladder with SVF are

Table 2. Compactness of the representations

Explained variations $85 \% 95 \% 98 \%$

\begin{tabular}{cccc}
\hline Displacement & 6 & 11 & 16 \\
\hline Pole ladder SVF & 7 & 10 & 14 \\
\hline
\end{tabular}
mainly related to the orientation and the relative position of the tricuspid valve and the pulmonary valve. Figure 3 summarizes these results for the point-to-point displacement and pole ladder with SVF. We compared the compactness of the representations (Table 2) and found pole ladder with SVF a very good explanation. 


\subsection{Limitations}

Cardiac motion tracking establishes point-to-point correspondences between mesh vertices, available here to benchmark our method. For databases that do not have such correspondence, one option is to register the segmented surfaces to the template, with the framework of currents or varifold-based deformation, which provide elegant mathematical approaches to match surfaces and establish point-to-point correspondence. The limitation of the method notably lays in registration accuracy, but this is compensated by the stability and one-step transport structure of the algorithm.

\section{Conclusion}

We proposed a general scheme to perform statistical modeling of the temporal deformation of the heart, directly based on meshes. The extensions of the pole ladder for parallel transport to mesh deformations were stable and accurate, of importance for the assessment of pathological changes. They also provided a more compact and interpretable representation that is useful for statistical modeling, and prevented surface folds in the range of population variability.

The method is adaptable to other anatomies with temporal or longitudinal data. Perspectives notably include synthetic experiments; the possibility to simulate physiologically-realistic motion on a given subject's anatomy, of relevance to better understand shape and motion interactions, or inversely to generate template sequences derived from populations, to be used for physiological modeling.

Acknowledgments. Part of the research was funded by the Agence Nationale de la Recherche (ANR)/ERA CoSysMed SysAFib project. The authors would like to thank fellows from Hôpital Pasteur, CHU de Nice, France for preparing the data.

\section{References}

1. Bai, W., Shi, W., de Marvao, A., Dawes, T.J., ORegan, D.P., Cook, S.A., Rueckert, D.: A bi-ventricular cardiac atlas built from 1000+ high resolution mr images of healthy subjects and an analysis of shape and motion. Medical image analysis 26(1), 133-145 (2015)

2. Bossa, M., Hernandez, M., Olmos, S.: Contributions to 3d diffeomorphic atlas estimation: application to brain images. In: International Conference on Medical Image Computing and Computer-Assisted Intervention. pp. 667-674. Springer (2007)

3. Davis, M.H., Khotanzad, A., Flamig, D.P., Harms, S.E.: A physics-based coordinate transformation for 3 -d image matching. IEEE transactions on medical imaging 16(3), 317-328 (1997)

4. Duchateau, N., De Craene, M., Piella, G., Silva, E., Doltra, A., Sitges, M., Bijnens, B.H., Frangi, A.F.: A spatiotemporal statistical atlas of motion for the quantification of abnormal myocardial tissue velocities. Medical image analysis 15(3), 316-328 (2011) 
5. Gavrilov, A.V.: Algebraic properties of covariant derivative and composition of exponential maps. Matematicheskie Trudy 9(1), 3-20 (2006)

6. Lorenzi, M., Ayache, N., Pennec, X.: Schilds ladder for the parallel transport of deformations in time series of images. In: Biennial International Conference on Information Processing in Medical Imaging. pp. 463-474. Springer (2011)

7. Lorenzi, M., Pennec, X.: Efficient parallel transport of deformations in time series of images: from schilds to pole ladder. Journal of Mathematical Imaging and Vision 50(1-2), 5-17 (2014)

8. Louis, M., Bône, A., Charlier, B., Durrleman, S., Initiative, A.D.N., et al.: Parallel transport in shape analysis: a scalable numerical scheme. In: International Conference on Geometric Science of Information. pp. 29-37. Springer (2017)

9. Moceri, P., Duchateau, N., Baudouy, D., Schouver, E.D., Leroy, S., Squara, F., Ferrari, E., Sermesant, M.: Three-dimensional right-ventricular regional deformation and survival in pulmonary hypertension. European Heart Journal-Cardiovascular Imaging (2017)

10. Pennec, X.: Parallel transport with pole ladder: a third order scheme in affine connection spaces which is exact in affine symmetric spaces. arXiv preprint arXiv:1805.11436 (2018)

11. Qiu, A., Younes, L., Miller, M.I., Csernansky, J.G.: Parallel transport in diffeomorphisms distinguishes the time-dependent pattern of hippocampal surface deformation due to healthy aging and the dementia of the alzheimer's type. NeuroImage 40(1), 68-76 (2008)

12. Rohé, M.M., Datar, M., Heimann, T., Sermesant, M., Pennec, X.: Svf-net: Learning deformable image registration using shape matching. In: International Conference on Medical Image Computing and Computer-Assisted Intervention. pp. 266-274. Springer (2017)

13. Vercauteren, T., Pennec, X., Perchant, A., Ayache, N.: Symmetric log-domain diffeomorphic registration: A demons-based approach. In: International conference on medical image computing and computer-assisted intervention. pp. 754-761. Springer (2008)

14. Younes, L.: Jacobi fields in groups of diffeomorphisms and applications. Quarterly of applied mathematics pp. 113-134 (2007) 